\title{
ACCESSIBILITY MODELS FOR NATURAL RESOURCE MANAGEMENT
}

\author{
Mauricio Xavier González Mantilla ${ }^{1}$, Dick van der Zee $e^{2}$ \\ ${ }^{1}$ International Institute for Aerospace Survey and Earth Sciences (ITC), P.O.Box 6, 7500 \\ AA Enschede, the Netherlands. E-mail:vanderzee@itc.nl \\ ${ }^{2}$ Universidad Nacional Autonoma de Honduras, Department of Geographic Information \\ Science and Technology. E-mail: mauriciog78jn@yahoo.com
}

Received: $20^{\text {th }}$ November 2009, Accepted: $8^{\text {th }}$ March 2010

\begin{abstract}
Accessibility is an important aspect in the evaluation of suitability of an area in the context of natural resource management or landscape ecology. In many geographic information software packages a distance function is included, but the creation of adequate accessibility models requires more than simple distance calculation and in practice is not that easy. A theoretical test area is used to demonstrate how different terrain variables will influence the results of the application of the distance function. With the understanding obtained that way, the approach is applied in a real case study area to illustrate its possibilities but also some difficulties and limitations that are still encountered.
\end{abstract}

Key words: Accessibility, distance, travel time, GIS, resistance factor

\section{INTRODUCTION}

The aspect of accessibility should play an important role in many Natural Resource Management or Landscape Ecology studies, especially when trying to evaluate the suitability of an area for a specific purpose or to assess the possible impact of an anticipated or planned interference.

But in many cases such evaluations are very elaborate in the analysis of the physical aspects of soil and terrain, but rather ephemeral in dealing with the accessibility factor. Generating and displaying buffer zones of a certain distance around settlements and/or along roads, often disregarding physical obstacles, is commonly encountered. See for example Figure 1, in which the distance zones ignore the presence of a lake.

As a first approximation this may be acceptable, but for more refined analysis more detail is required. The present generation of Geographic Information Systems (GIS) does allow such detailed analysis. Where the vector based systems seem to be confined to the buffer zone approach, the raster based systems can support more sophistication. The aim of this paper is to indicate in what way the distance function of GIS can be used to create more or less realistic accessibility models. 
Fig. 1: Representation of a study area with indication of distance zones from roads and settlements. (Valenzuela, 1988)

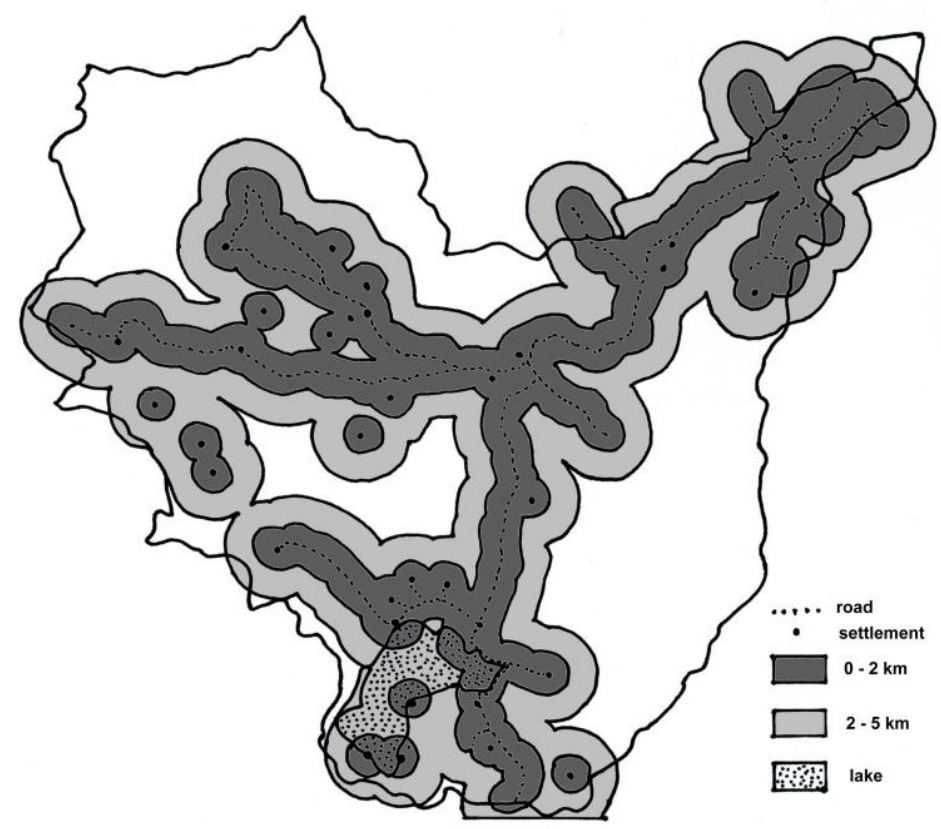

\section{UNDERSTANDING THE BASICS OF ACCESSIBILITY MODELLING}

Most GISs have a facility to calculate distances from a certain point or line. The procedure and terminology may vary from one system to the other, but the basic principles remain the same.

For using the distance facility the point, line or area from which the distance has to be calculated should be defined as "origin" or "target". Positive values can be assigned to the rest of the area in order to give weights, and negative values to demarcate areas that are unreachable. In this way a "resistance" or "cost" surface is created.

The software then calculates values in meters for the defined setting (in some systems may be still in grid cells) from the origin. (Zee, 2008)

Visualization of these distances usually calls for classification. This can be done, for example, by dividing the scores for meters by thousand to obtain the scores in kilometres, or by using a conversion table or utility to gain a similar result.

To understand how the characteristics of a resistance surface will influence the shape and width of distance zones it is worthwhile to apply it on a theoretical test area first. As an example an imaginary area of $10 \times 10 \mathrm{~km}$ with one central city was digitized and then converted to a raster map with grid cells of 25 meters, in which the city was defined as the origin.

Having assigned the value of 1 to the surrounding area, application of the "distance function" of GIS creates a nice set of concentric distance zones that could be easily classified into kilometre zones, as is shown in figure 2 . 
Fig. 2: Distance zones around one area of origin, classified in kilometres

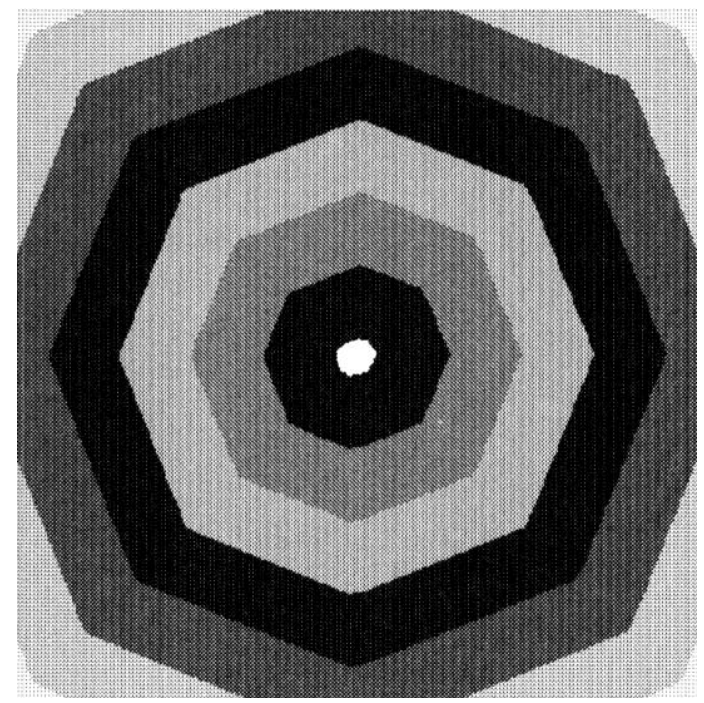

To add one complexity, the uniform area around the city has been subdivided into three zones with values of 1, 2, and 3 respectively assigned to them, representing various degrees of resistance to traffic (of any kind) as can be offered, for example, by various cover types, terrain or slope conditions. This situation is represented in Figure 3 (left). Applying the distance function to this situation and classifying the results again into kilometre zones gives the result as shown in Figure 3 (right).

Fig. 3: left: Central city with three zones of different resistance (black $=1$; medium grey $=2$; light grey $=3$ ); right: The distance zones resulting from this situation classified in kilometres.
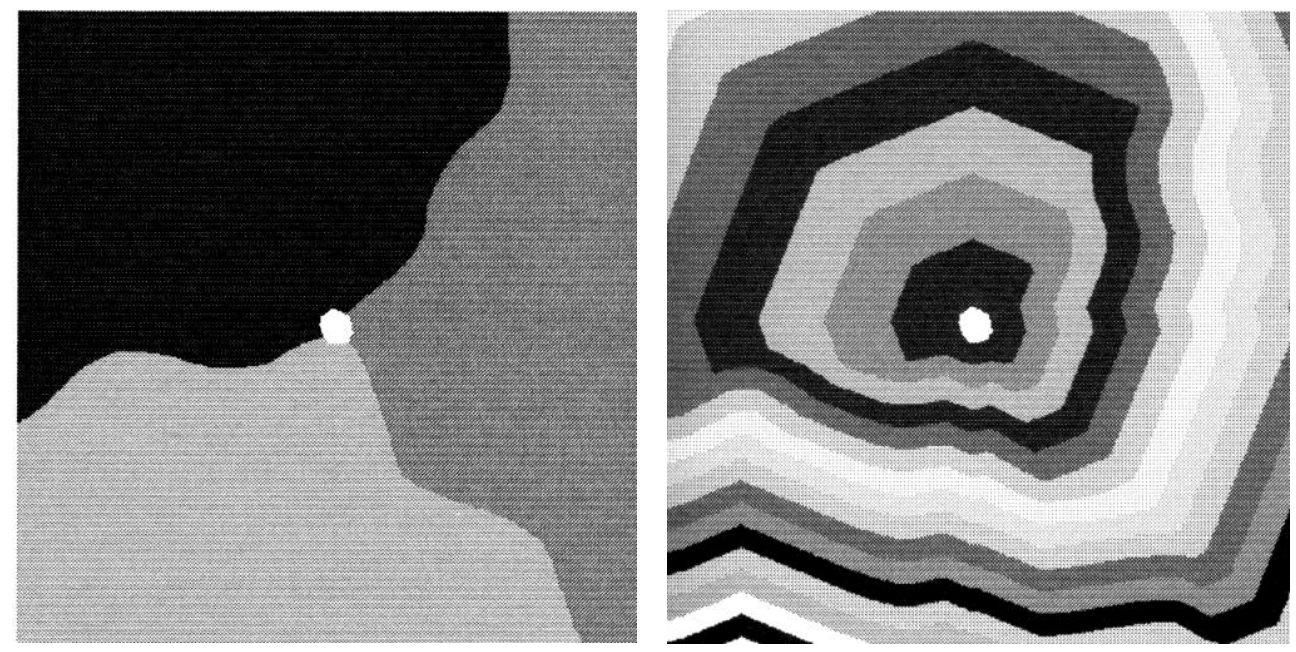
In the area of the value of 1, the zones are of the same width as those in Figure 2. Where the value is 2 , the zones are half the width. Where the value is 3 , the zones are one third of the width. Of course, it has to be realized that now the zones do not represent real distances any more, but should rather be expressed in the equivalent of travel time.

Such a conversion has to be based on empirical or experimental data - indicating how much a certain terrain condition will slow down traffic - rather then by rule of thumb. It should also take into account the way of transport. Therefore, considering an average walking speed of 6 kilometres per hour, the zones in figure 3 (right) would correspond to intervals of 10 minutes travel time. (Zee, 2008)

In another example one isolated zone with different resistance is added to the otherwise homogeneous area. In one case this zone was considered to be a mountain barrier and given a high resistance value (50). Using the same classification as for Figure 2 the results of applying the distance function in this case are as shown in Figure 4 (left). The distance zones curve around the mountain and penetrate it only with difficulty. The effort it takes is represented by the very narrow zones. When playing with the resistance value in the model it becomes very clear that with increasing height of this value the zones become narrower and narrower.

Should the zone be defined as completely inaccessible, for example a lake rather than a mountain, application of the distance function results in the same pattern of zones curving around the obstacle as in Figure 4 (left), but the obstacle itself remains a completely blank area, see Figure 4 (right).

Fig. 4: left: Central city in homogeneous area with one isolated resistance zone (mountain barrier) and the corresponding distance/travel time zones; right: The same but then with an isolated absolutely inaccessible obstacle area (lake).
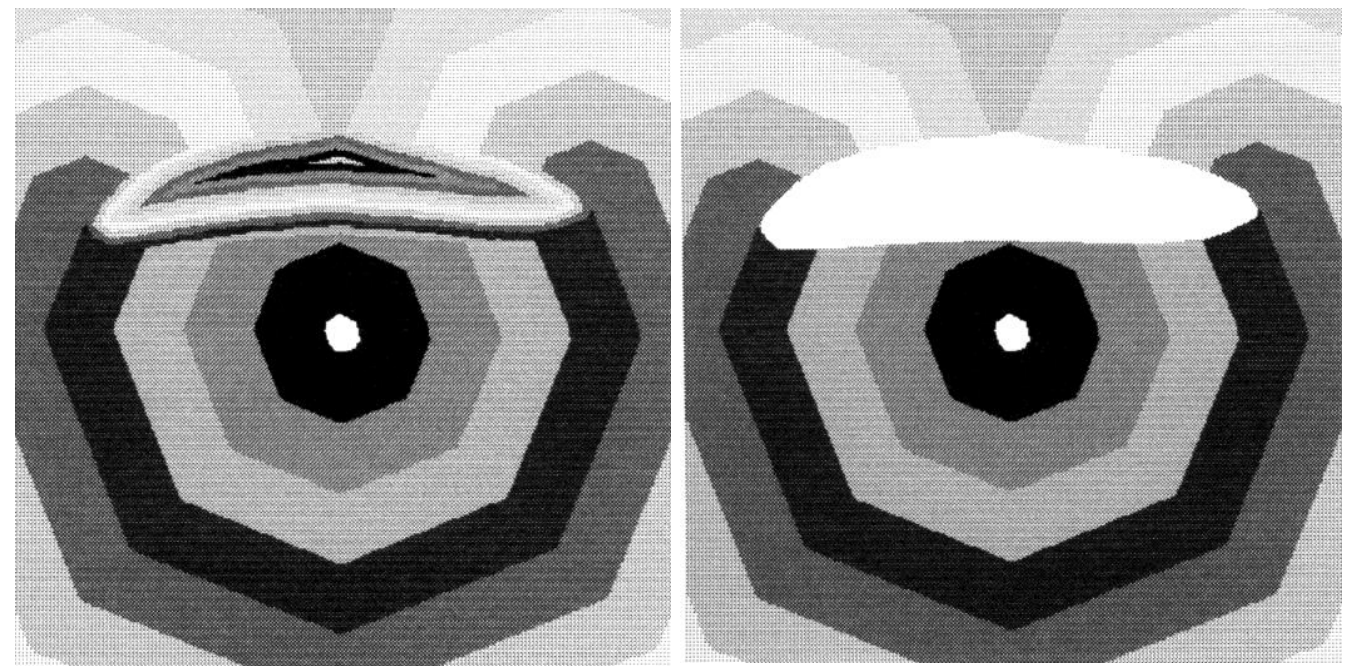

It may not be too difficult to visualize what would happen to the distance zones if the completely inaccessible area was represented by a river crossing the entire area of study, rather than a lake, and then, what would be the effect of creating one place where it is possible to cross that barrier. (Zee, 2008) 
In the previous models the access was more or less thought of in terms of cross-country accessibility. Traffic commonly follows roads and other lines of infrastructure. For some vehicles the accessibility is restricted to these lines and everything else would be inaccessible. But resistance values can also be used in this case to express the difference in time needed to cover the same distance with a vehicle along the road, or by foot cross-country. For example, if on the road the average speed was 60 kilometres per hour, and by foot it is 5 kilometres per hour, a resistance value relation of 12:1 should be applied.

Thus, in the resistance surface the road gets the value 1 and the surrounding area the value 12. Although some vehicles can also travel off road, be it at lower speed, the thought behind adding the value 12 is to show the effect of the combination of travelling by vehicle along the road and then continuing off the road on foot.

In a further complication of the infrastructural setting, secondary roads with the value 2 , representing the average travel speed of 30 kilometres per hour, are introduced; see Figure 5 (left). The resulting accessibility zone pattern, expressed in zones of 5 minutes travel time, is displayed in Figure 5 (right). The entire area is not covered by zones because the upper threshold limit has been reached, beyond which no values are displayed. This can be fixed by adapting the scale factor or the classification range. (Zee, 2008)

Fig. 5: left: Central city in homogeneous area with primary (solid line) and secondary (dashed line) roads; right: the resulting pattern of accessibility zones in intervals of five minutes travel time.
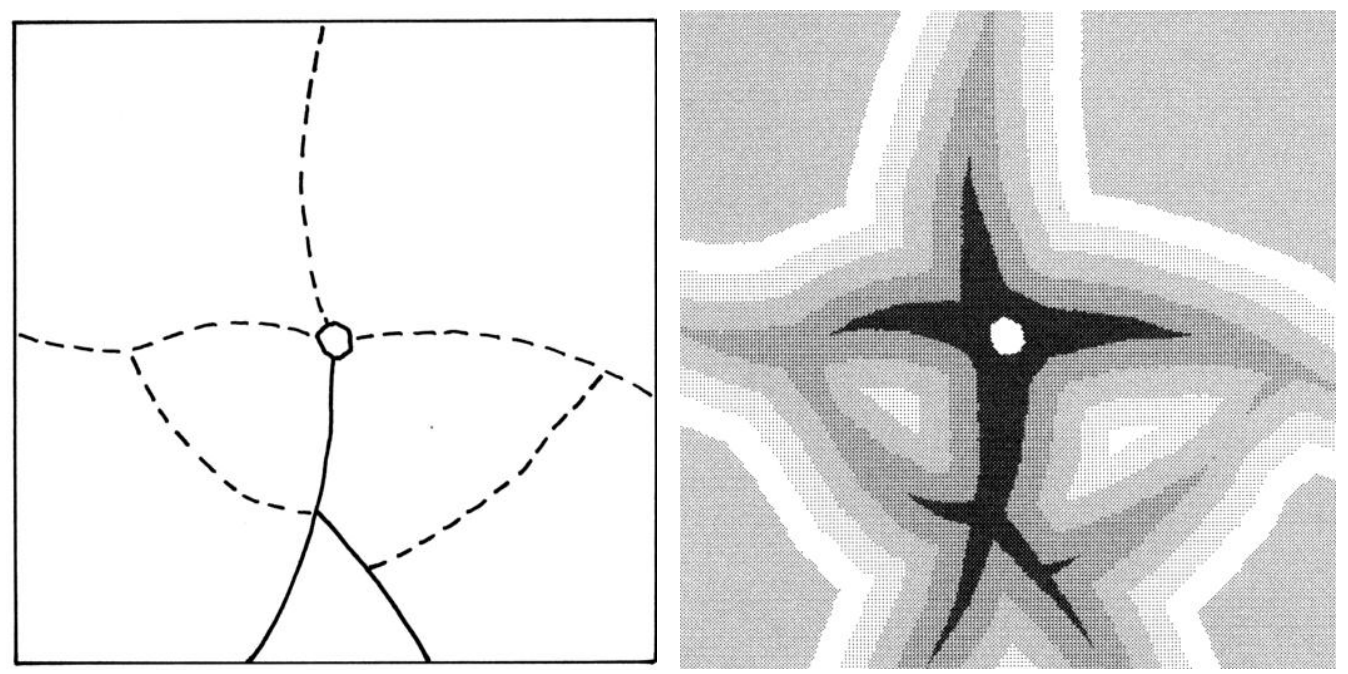

When being aware of the effect of each of the individual factors on the distance function, it is time to see how these factors act while combined in a more or less realistic situation. To do so first a map layer is created containing a city (defined as origin), a river (absolute obstacle) and a mountain (high resistance value), with the remaining area of the value of 12 . On top of that is superimposed the map layer containing main roads (value 1) and secondary roads (value 2). The section of secondary road crossing the mountain has been re-digitized to allow for the curving pattern a mountain-road is supposed to have. See Figure 6 (left). 
Fig. 6: left: Central city in with primary and secondary roads, a river as obstacle and a mountain barrier; right: the resulting pattern of accessibility zones in intervals of ten minutes travel time.
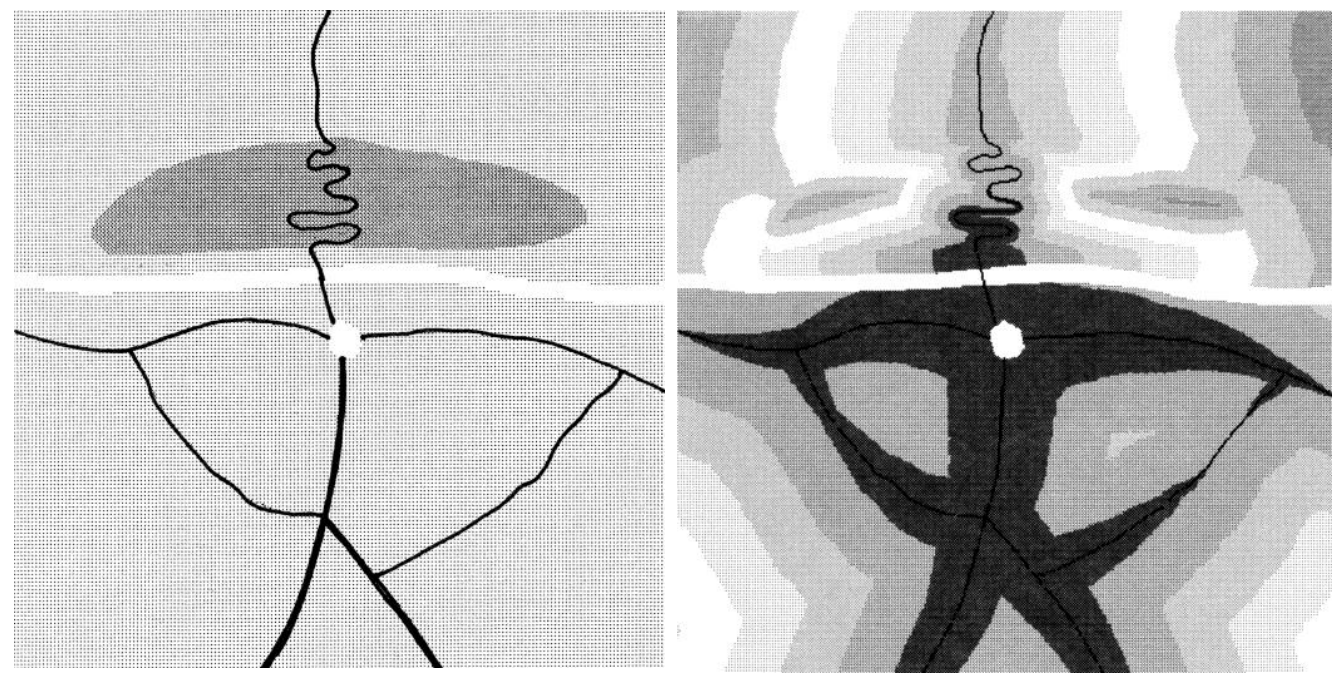

Applying the distance function to the combined map, using the appropriate scale to eliminate the upper threshold in the classification and defining intervals corresponding to 10 minutes travel time, gave the result shown in Figure 6 (right).

Removing the threshold also allows to show zoning in more remote corners, as can be observed when comparing Figure 6 (right) b to Figure 5 (right). The pattern of zones south of the river is similar to that in figure 5 (right). To the north of the river the pattern is influenced firstly by the fact that the river can only be crossed at one place, and secondly by the high resistance factor of the mountain.

The zones spreading out from the road that crosses the mountain are narrow. The wider zones spreading in front of the mountain and starting to curve around it, meet with the zones spreading from the road beyond the mountain, leaving some islands of high inaccessibility on the top of the mountain range on both sides of the mountain road.

In this way the various factors influencing accessibility can be combined in order to obtain a more or less realistic picture of the area's accessibility. Of course, the example has been kept rather simple, with only a few parameters. But in principle it can be expanded to include more refined weight factors, for example, by deriving resistance values from a Digital Elevation Model.

For such weight factors to become realistic, empirical data on distances covered by different modes of transport under different conditions have to be collected and validated. It might be useful to create accessibility models for different modes of transport separately first and only when relevant try to integrate or combine them into one overall accessibility model. Accessibility may also have to be addressed for different seasons: winter and summer or wet and dry season. (Zee, 2008)

A lot of research still has to be done before accessibility models can really be operational. But, when using accessibility models in this way, even if still only by rule of thumb, it may be relatively simple to assess the influence of construction of a new road, or the effect of the choice of a particular route for it. It may also be assessed in this way whether certain 
natural resources are under pressure because of their relative accessibility and whether certain measures to restrict accessibility would have the desired effect. A real life case study may serve to illustrate a practical application.

\section{APPLICATION OF ACCESSIBILITY ANALYSIS}

An early attempt to apply this approach of accessibility modelling was hampered by the fact that the digital elevation model and the digital land cover map were not available, which resulted in manually plotted travel time zones (Zee, 1989). Other examples have been elaborated in several case studies, for example, one related to forest degradation and deforestation in relation to accessibility (Malyvanh, 1998), another trying to relate the number of reported malaria cases not only to the areas with high suitability for the malaria mosquito, but also to the accessibility to hospitals and polyclinics (Ohemeng, 1997). However, in these examples the resistance factors were based on mere assumptions. In the very nicely elaborated example that has been presented by Mauricio González for the Ruvu North Forest Reserve in Tanzania, just north of Dar es Salaam, (González Mantilla, 2007) the assumptions have been based on field observations and empirical data. Therefore this case will be used to illustrate the possibilities of accessibility modelling. The accessibility analysis approach was applied to assess the vulnerability of the resources in this forest reserve to the pressure exerted on them from the surrounding villages. The mode of transport considered was walking, because although people tend to use bicycles to get into the area, they walk back pushing their bikes with a load of charcoal or fuel wood. The use of trucks is still exceptional.

The different variables included in the accessibility model were the availability and condition of roads, land cover classes, slope steepness and the location of settlements.

The location of the settlements that were defined as points of origin for the calculation of the accessibility model was extracted from the existing topographic maps from 1987 . The road network was digitized from the same topographic map and was (partly) updated during the fieldwork in 2007 using the Global Positioning System (GPS) equipment. The roads were classified into five types depending on their quality and for each type the travelling speed in kilometres per hour was assessed, partly based on experiences during the fieldwork. The travelling speed by car was considered too, in order to indicate the influence this mode of transportation might have. See Table 1.

Table 1: Travelling speed per road type

\begin{tabular}{|l|l|c|c|}
\hline \multirow{2}{*}{ Road type } & \multicolumn{2}{c|}{ Road Quality } & \multicolumn{2}{c|}{ Travelling speed in km/h } \\
\cline { 3 - 4 } & & By walking & By car \\
\hline Main road & All weather road: bound surface & 6 & 60 \\
\hline Secondary road I & All weather road: loose surface & 6 & 50 \\
\hline $\begin{array}{l}\text { Secondary road } \\
\text { II }\end{array}$ & Dry weather road & 6 & 30 \\
\hline Track & & 4 & 20 \\
\hline Trail & Main track (motorable) & 3 & 0 \\
\hline
\end{tabular}

With regard to the fact that not all area inside the reserve is included in the road network, the influence of the land cover on accessibility has to be considered. During the fieldwork it 
was observed that walking through thicket was more difficult than walking through grassland or woodland. A land cover map made on the base of a SPOT image from 2003 was used for the analysis of this aspect. For the five relevant cover types the average travelling speed has been assessed based on fieldwork experience. See Table 2.

Table 2: Travelling speed per land cover class

\begin{tabular}{|l|c|}
\hline Land cover class & Travelling speed in $\mathbf{k m} / \mathbf{h}$ \\
\hline Thicket & 0.5 \\
\hline Shrubland & 0.7 \\
\hline Woodland & 1.0 \\
\hline Grassland & 2.0 \\
\hline Agricultural land/bare soil & 3.0 \\
\hline
\end{tabular}

Once the travel speed classifications for land cover classes and road types were translated into map layers, the two layers could be merged so that values for the roads are incorporated in the land cover layer.

In addition, slope steepness is a variable that also may influence the speed of travel. The slope steepness was derived from the available Digital Elevation Model (DEM). The slopes were classified into 5 classes and to each class a factor was attributed that represented the reduction in travelling speed related to the slope percentage. See Table 3. These slope speed factors are partly based on field experience, but mainly on estimated guesses.

Table 3: Travelling speed factor per slope class

\begin{tabular}{|c|c|}
\hline Slope \% & Slope speed factor \\
\hline 0 & 1.0 \\
\hline $0-2$ & 0.9 \\
\hline $3-4$ & 0.8 \\
\hline $5-6$ & 0.6 \\
\hline $7-10$ & 0.5 \\
\hline
\end{tabular}

To apply the influence of the slope, the composite map with travel speed values for land cover and road types was multiplied by the values gained from the slope factor map. The result is a travel speed map.

In order to obtain a travel condition map, travel cost surface or resistance surface; the values of the travel speed map have to be inversed. This is done by dividing the travel speed under normal conditions $(=6 \mathrm{~km} / \mathrm{h})$ by the values on the travel speed map. For example, when the pixel value of the travel condition map is 1 there is no resistance, so the walking speed is assumed to be $6 \mathrm{~km} / \mathrm{h}$. If the pixel value is 2 the travel speed will be 3 $\mathrm{km} / \mathrm{h}$. Therefore, the higher the values on the travel condition map, the longer it will take to reach a certain point. The resulting travel condition map is presented in Figure 7. 


\section{Fig. 7: Travel Condition Map of the Ruvu North Forest Reserve (González Mantilla,} 2007)

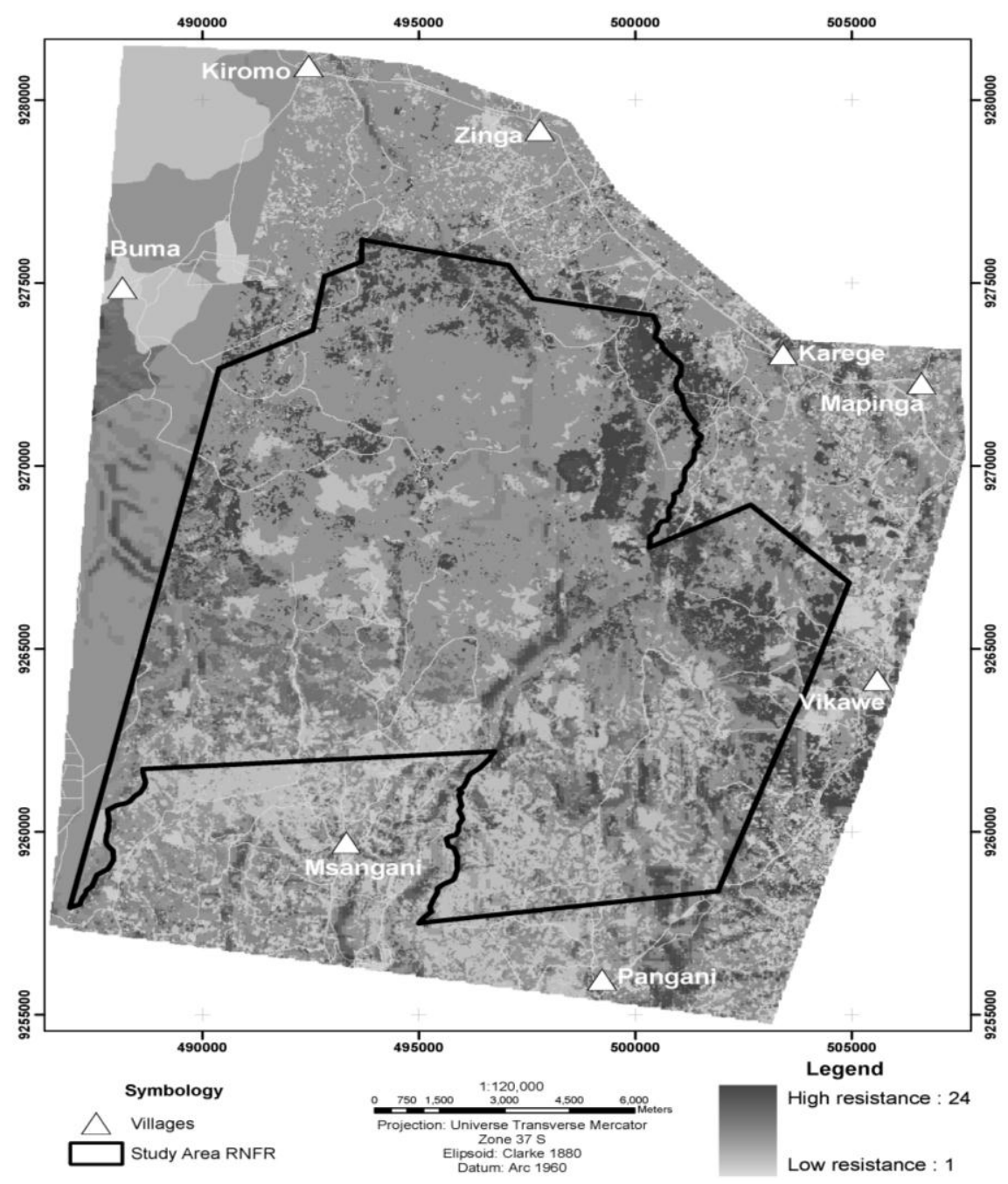

Note that the western part of the study area was not covered by the SPOT image, therefore the land cover aspect of the accessibility could not be included there, instead, an average value had to be used. Because of the restricted area covered by the satellite, originally only the eastern part of the Ruvu North Forest Reserve was included as the study area. A small expansion to the west was required to overcome some problems in the accessibility model as will be explained in the following.

Before arriving to the final result of accessibility analysis, different models were used and compared to better appreciate which method applied was the most appropriate in order to generate travel time from the villages to the study area. One of the methods just considered a straight distance calculation from the settlements to the reserve without any resistance (Figure 8a). As can be observed, the highest value of travel time is 1.5 hours, which does not represent the real situation in the area. (González Mantilla, 2007) 
Fig. 8: Results of the distance modelling without considering any resistance factor (a), considering resistance factors only for the area covered by satellite image, ignoring the road coming in from the west (b), and taking into account the presence of Buma (c).
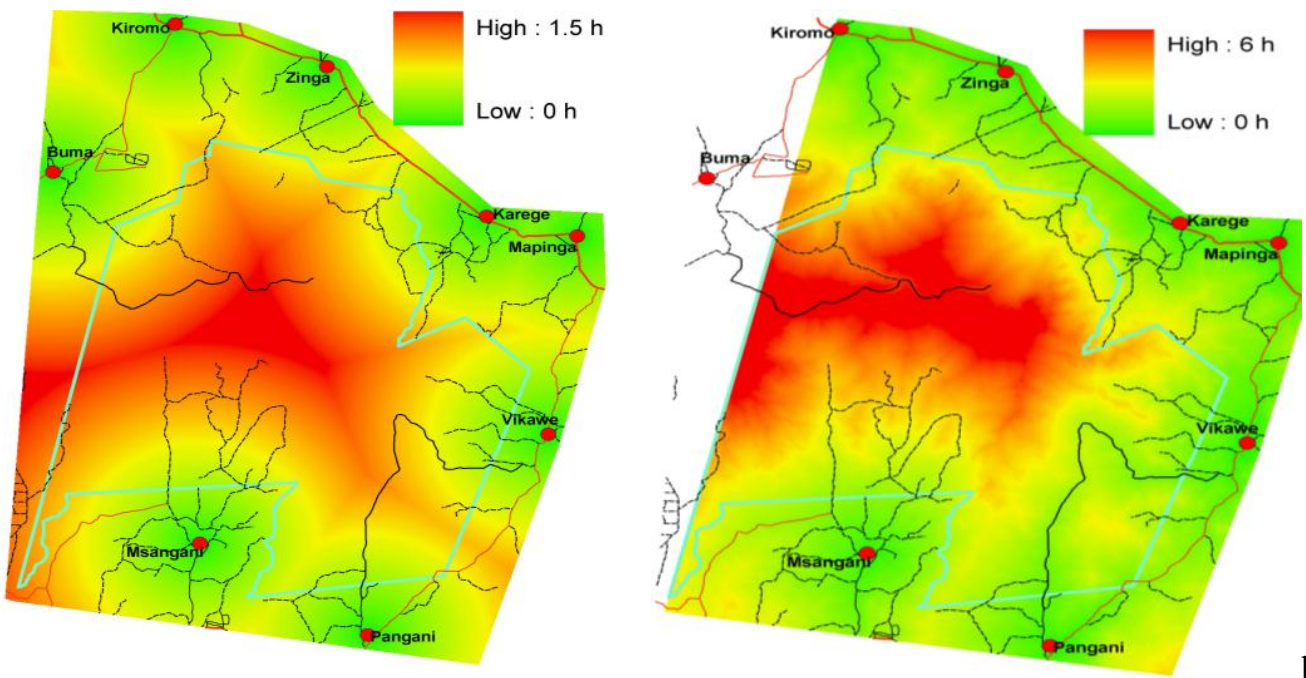

a

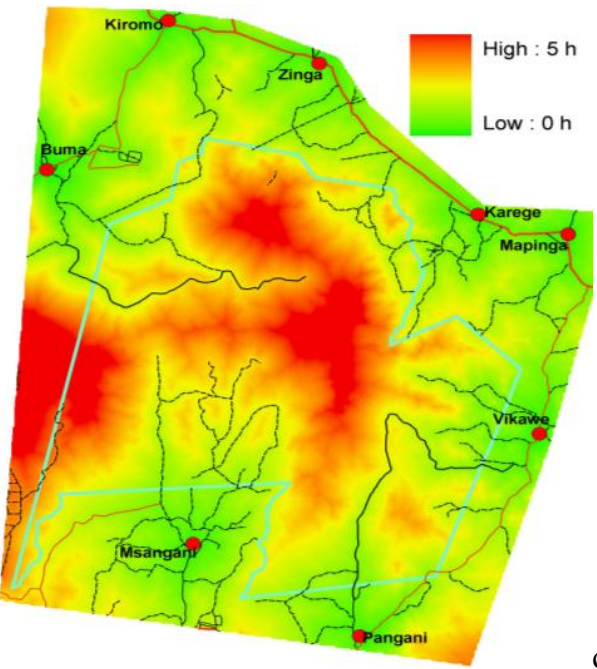

NB: High = high resistance therefore $=$ low accessibility!

Another one was generated excluding the Buma village in the north-west of the study area. (Figure $8 \mathrm{~b}$ ). The reason to do so was the fact that originally the accessibility model was only generated for the area that was covered by the available satellite image from which the cover types were derived. But as a result the influence of the location of Buma, and of the road that leads from it into the reserve, is not represented. Therefore, this result does not permit to appreciate the magnitude of the intervention in this part of the study area. 
Fig. 9: Travel Time Zones Map of the Ruvu North Forest Reserve (González Mantilla, 2007)

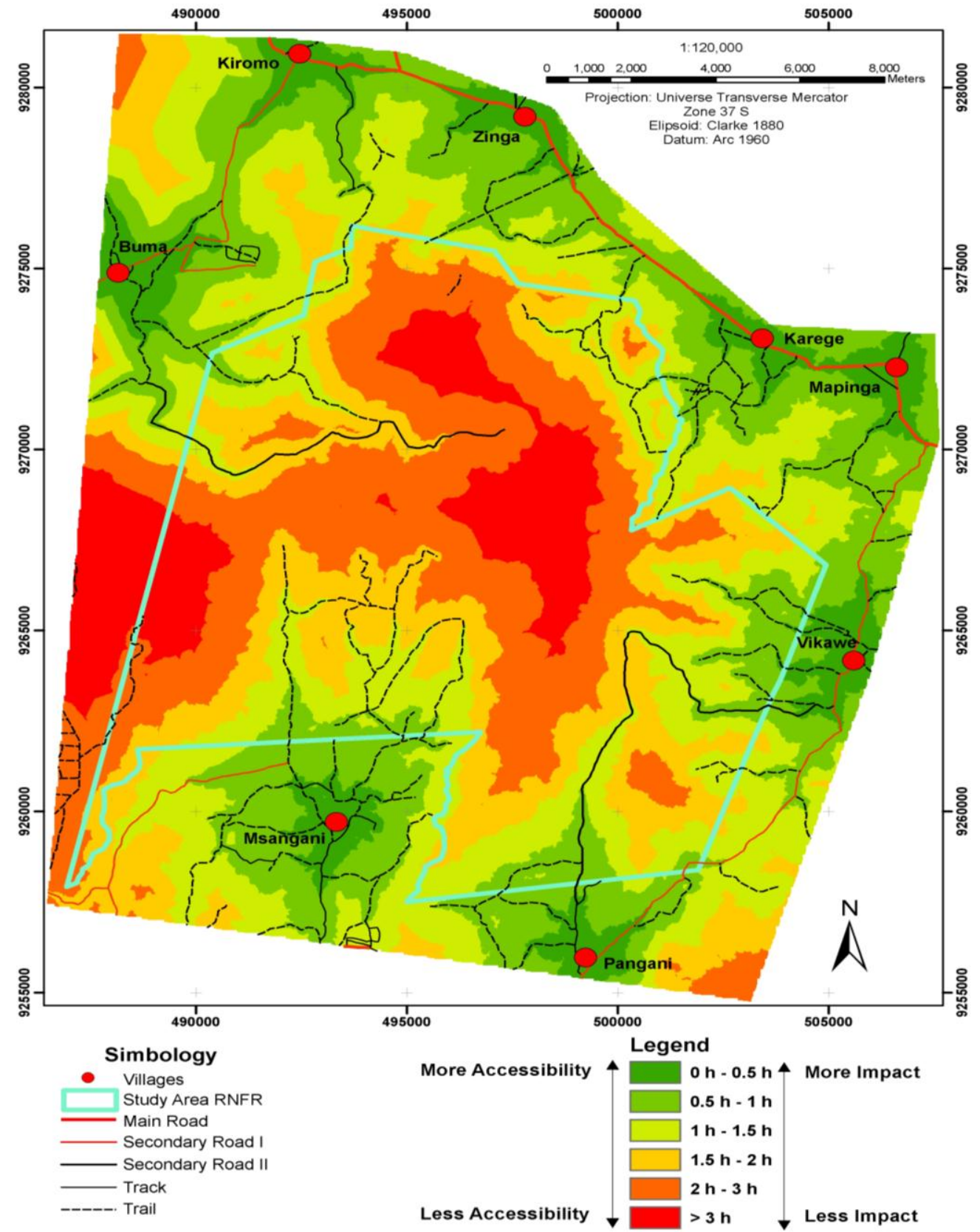

The travel condition map has been expanded accordingly, taking an average cover resistance value for the area of which no actual cover data were available. When applying this expanded travel conditions map to the accessibility analysis (Figure 8c), both the 
resistance of the terrain and the influence of the location of all the settlements around the study area are considered. Therefore, this result was considered to be the most appropriate one for the spatial management plan of the RNFR. (González Mantilla, 2007). Should the data beyond the boundary not have been available at all this would be a serious problem for a realistic accessibility analysis.

Applying a classification to the continuum of values in Figure 8c, including both the resistance value from the travel conditions map as well as the influence of the location of all the settlements around the study area, resulted in the final accessibility map (Figure 9) or the travel time zones map. It gives a clear impression of which areas are under great pressure, and where restrictions to accessibility, if properly enforced, could be most effective.

\section{CONCLUSiOnS}

The distance function in many GIS software packages can be very well used for accessibility modelling. The resistance factors of the terrain or landscape need to be defined in as much detail as possible on basis of empirical data rather than on mere assumptions, and distances have to be translated into travel time for the models to become close to reality Even then the results should be critically examined for inconsistencies or anomalies resulting from the possibility of overlooking certain aspects such as influences from outside of the case study area. However, when taking all this into consideration, there is a great potential for creating accessibility models not only for all kinds of human traffic or travel, but also for animal movements and maybe even plant dispersal.

\section{REFERENCES}

González Mantilla, M. (2007). Spatial Management Plan for the Ruvu North Forest Reserve, Tanzania. Individual Final Assignment (IFA) report, ITC, Enschede, the Netherlands.

Malyvanh, M. (1998). Land Use Change and Accessibility. Unpublished MSc Thesis Wageningen University and ITC, Enschede.

Ohemeng, F.D. (1997). Modelling the spatial distribution of Anopheles mosquito for malaria risk zoning using remote sensing and GIS. A case study in the Zambesi basin of Zimbabwe. Unpublished MSc thesis, ITC, Enschede, the Netherlands.

Valenzuela, C.R. (1988). ILWIS overview. ITC Journal, 1988(1): 4 - 14.

Zee, D.V.D. (1989). The importance of the spatial aspect in the evaluation of recreational resources in the landscape. Ekológia (CSSR), 8(2): 143-154.

Zee, D.V.D. (2008). Accessibility models. Lecture note, Masters course in Natural Resource Management, Module 8: Scenarios and Modelling. ITC, Enschede, the Netherlands 